CUQM-118

math-ph/0610008

\title{
Wave equation and dispersion relations for a compressible rotating fluid
}

\author{
José Marín-Antuña ${ }^{\dagger}$, Richard L. Hall ${ }^{\ddagger}$, and Nasser Saad* \\ $\dagger$ Department of Theoretical Physics, Faculty of Physics, University of Havana. \\ ${ }^{\ddagger}$ Department of Mathematics and Statistics, Concordia University, 1455 de \\ Maisonneuve Boulevard West, Montréal, Québec, Canada H3G 1 M8 \\ *Department of Mathematics and Statistics, University of Prince Edward Island, \\ 550 University Avenue, Charlottetown, Prince Edward Island, Canada C1A 4 P3
}

\begin{abstract}
A fundamental non-classical fourth-order partial differential equation to describe small amplitude linear oscillations in a rotating compressible fluid, is obtained. The dispersion relations for such a fluid, and the different regions of the group and phase velocity are analyzed.
\end{abstract}

Key words: Rotating fluids, small waves, dispersion relations.

PACS: 03.65.Ge

\section{Introduction}

In this paper we study the dynamics of a rotating compressible fluid, taking into account such factors as non-homogeneity, and the presence of Coriolis forces due to the Earth rotation. The work is devoted principally to the study of one of these aspects of the problem, namely the influence of the rotation over the wave propagation in the fluid.

Much work has been devoted to theoretical, mathematical, and physical aspects of waves in compressible and incompressible fluids. The literature of this

Email addresses: marin@fisica.uh.cu, rhall@mathstat.concordia.ca, nsaad@upei.ca (José Marín-Antuña ${ }^{\dagger}$, Richard L. Hall ${ }^{\ddagger}$, and Nasser Saad*). 
subject is enormous, primarily because it provides a basis for a wide range of applications and techniques, including the solution and modeling of Geophysical situations, and in the theory of stars. For example the papers [1]-[4], and books which discuss the fundamental equations governing waves in such fluids [5]. More particularly, study of problems of wave propagation in a rotating compressible fluid have been the subject of several earlier publications [6] [7]. Some works have been devoted to the general theory of equations in stratified and rotating fluids [8] - [10]. Some monographs are devoted to this type of problem [11] - [13]. Nevertheless, with few exceptions [14], the systematic analtytic study of concrete non-stationary problems of wave propagation in a rotating compressible fluid have not been treated.

The present line of investigation has its origin in the seminal work of S. L. Sobolev [15]. Starting from the hydrodynamic equations we obtain in Sec. 2 a non-classical fourth-order partial differential equation, which describes small amplitude linear oscillations in such a fluid. In Sec. 3 we analyse the dispersion relations for linear waves in the fluid, and discuss the different numerical regions of the group and phase velocities of the propagating waves, including their dependence on the direction of the wave vector with respect to the rotation axis of the fluid.

The results obtained are potentially useful for the study of the structure of the hydrophysical fluid fields in oceans and in the Earth atmosphere.

\section{Basic equations}

Consider an ideal compressible fluid that fills the whole space. We assume that the fluid rotates with constant velocity $\alpha / 2$ around a given axis. The fluid motion is refered to a system of Cartesian coordinates $\vec{x}=\left(x_{1}, x_{2}, x_{3}\right)$ which rotates with the fluid. The $O x_{3}$ axis is directed along the rotation axis.

For such conditions, following ideas developed in [11] and [12], the system of hydrodynamic equations which describe the fluid is given by the following:

(1) The Euler equation:

$$
\frac{\partial \vec{v}}{\partial t}+(\vec{v} \cdot \vec{\nabla}) \vec{v}+\frac{1}{\rho} \vec{\nabla} P-\vec{f}=0
$$

where $\vec{v}=\left(v_{1}, v_{2}, v_{3}\right)$ is the vector velocity of the fluid particles, $P$ is the

pressure, $\rho$ is the density of the fluid, and $\vec{f}$ is the force per unit mass acting on the fluid. 
(2) The continuity equation

$$
\frac{d \rho}{d t}+\rho(\vec{\nabla} \cdot \vec{v})=0
$$

(3) The Thermodynamic state equation

$$
P=P(s, t)
$$

where $s$ is the entropy of the fluid.

If we denote $\left(\frac{\partial P}{\partial \rho}\right)_{s}$ by $c^{2}$, that is to say

$$
c^{2}=\left(\frac{\partial P}{\partial \rho}\right)_{s}
$$

then we have

$$
\frac{d P}{d t}=c^{2} \frac{d \rho}{d t},
$$

where $c^{2}$ is a function of $P$ and $\rho$, and $c$ has the meaning of the sound velocity in the fluid. Taking into account (5), Eq. (2) can be written

$$
\frac{1}{c^{2}} \frac{d P}{d t}+\rho \vec{\nabla} \cdot \vec{v}=0
$$

for iso-entropic motions in the fluid. In (1) $\vec{f}$ is the Coriolis force due to the rotation of the fluid. This means

$$
\vec{f}=-\vec{\alpha} \times \vec{v}
$$

The corresponding Euler equation now becomes

$$
\frac{\partial \vec{v}}{\partial t}+(\vec{v} \cdot \vec{\nabla}) \vec{v}+\frac{1}{\rho} \vec{\nabla} P+\vec{\alpha} \times \vec{v}=0 .
$$

Eq. (8) is nonlinear. Therefore, the exact theory of physical processes in the fluid is nonlinear. In this paper, we study a linear approximation, and, to this end, we consider small perturbations in the fluid. We therefore linearize equation (8). The nonlinear term in (8) is the inertial one, $(\vec{v} \cdot \vec{\nabla}) \vec{v}$. We let 
$v_{0}$ be the velocity amplitude, $\gamma$ the frequency of the wave, and $k$ the wave number. Then, we have

$$
\varepsilon=\frac{(\vec{v} \cdot \vec{\nabla}) \vec{v}}{\frac{\partial \vec{v}}{\partial t}}=\frac{k v_{0}^{2}}{\gamma v_{0}}=\frac{v_{0}}{v_{p h}},
$$

where $v_{p h}=\gamma / k$ is the phase velocity of the wave. $\varepsilon$ is a parameter of nonlinearity. We assume $\varepsilon \ll 1$, that means that the velocity of the fluid particles on the wave is much less than the phase velocity of the wave. Under these conditions, we can eliminate the inertial term and obtain

$$
\frac{\partial \vec{v}}{\partial t}+\vec{\alpha} \times \vec{v}+\frac{1}{\rho} \vec{\nabla} P=0
$$

This equation, along with (6), comprises the system of equations we shall study. We consider small displacements of the pressure and of the density with respect to an equilibrium values:

$$
P=p_{0}+p, \rho=\rho_{0}+\rho^{\prime}, p \ll p_{0}, \rho^{\prime} \ll \rho_{0} .
$$

Under these conditions (10) gives

$$
\frac{\partial \vec{v}}{\partial t}+\vec{\alpha} \times \vec{v}+\frac{1}{\rho_{0}+\rho^{\prime}} \vec{\nabla}\left(p_{0}+p\right)=0
$$

and (6) gives:

$$
\frac{1}{c^{2}} \frac{d\left(p_{0}+p\right)}{d t}+\left(\rho_{0}+\rho^{\prime}\right) \vec{\nabla} \cdot \vec{v}=0
$$

By neglecting $\rho^{\prime}$ in these expressions, we arrive at the linearized system of equations used to describe the small-amplitude motion in the fluid:

$$
\begin{aligned}
& \frac{\partial \vec{v}}{\partial t}+\vec{\alpha} \times \vec{v}+\frac{1}{\rho_{0}} \vec{\nabla} p=0, \\
& \frac{1}{c^{2}} \frac{d p}{d t}+\rho_{0} \vec{\nabla} \cdot \vec{v}=0
\end{aligned}
$$

where $p$ is the dynamical displacement of the pressure from the equilibrium position $p_{0}$. System (11) - (12) is a 4-equation system of first order, with 4-unknown variables, $p, v_{1}, v_{2}$, and $v_{3}$, which describes the small-amplitude motion (acoustic waves) in the ideal rotating fluid. 
We will consider what we will call 2D motion in the fluid: by this we mean variation of the dynamic pressure and of the velocity for which

$$
\frac{\partial p}{\partial x_{2}}=0
$$

and

$$
\frac{\partial \vec{v}}{\partial x_{2}}=0
$$

From the physical and geometrical points of view, these kinds of motion are possible only in domains with infinite cylindrical shape, with the generatrices parallel to the $O x_{2}$ axis. In what follows, we suppose that the main value of the density is given by $\rho_{0}=1$. Then, the system (11)-(12) in the variables $v_{1}$, $v_{2}, v_{3}$, and $p$ becomes

$$
\begin{aligned}
& \frac{\partial v_{1}}{\partial t}-\alpha v_{2}+\frac{\partial p}{\partial x_{1}}=0, \\
& \frac{\partial v_{2}}{\partial t}+\alpha v_{1}=0, \\
& \frac{\partial v_{3}}{\partial t}+\frac{\partial p}{\partial x_{3}}=0, \\
& \frac{1}{c^{2}} \frac{\partial p}{\partial t}+\frac{\partial v_{1}}{\partial x_{1}}+\frac{\partial v_{3}}{\partial x_{3}}=0 .
\end{aligned}
$$

From the system (13) - (16) it is straightforward to obtain the following equation

$$
L[u]=\frac{\partial^{2}}{\partial t^{2}}\left[\frac{1}{c^{2}} \frac{\partial^{2} u}{\partial t^{2}}-\nabla_{2}^{2} u+\frac{\alpha^{2}}{c^{2}} u\right]-\alpha^{2} \frac{\partial^{2} u}{\partial x_{3}^{2}}=0,
$$

where $\nabla_{2}^{2} \equiv \partial^{2} / \partial x_{1}^{2}+\partial^{2} / \partial x_{3}^{2}$ is the 2D Laplacian. Eq. (17) is satisfied by the pressure $p$ and also by the components of the velocity $v_{1}, v_{2}$, and $v_{3}$. This is a non-classical fourth order partial differential equation, which governs the waves in a rotating compressible fuid.

From (17) we can see that, when $\alpha=0$ (which means that the fluid does not rotate), the operator $L$ has the form $L=\frac{\partial^{2}}{\partial t^{2}}\left[\frac{1}{c^{2}} \frac{\partial^{2} u}{\partial t^{2}}-\nabla_{2}^{2} u\right]$, and therefore the solution must coincide with the solution of the $2 \mathrm{D}$ wave equation. This allows us to conclude that, as a result of the rotation of the fluid, some terms appear in the equation that take into account the rotation. The corresponding solutions reflect this fact by their dependence on the angular velocity $\alpha$. Moreover, when $\alpha \rightarrow 0$, the solutions of reduce to the well-known solutions for wave propagation in a compressible medium. 


\section{Dispersion relations}

In what follows we shall adopt units in which $c=1$. Thus

$$
L[u]=\frac{\partial^{2}}{\partial t^{2}}\left[\frac{\partial^{2} u}{\partial t^{2}}-\nabla_{2}^{2} u+\alpha^{2} u\right]-\alpha^{2} \frac{\partial^{2} u}{\partial x_{3}^{2}}=0 .
$$

We consider a solution of the form

$$
u=u_{0} e^{i(\vec{k} \cdot \vec{x}+\gamma t)}
$$

where $\vec{k}=\left(k_{1}, k_{3}\right)$ is the wave vector, $\vec{x}=\left(x_{1}, x_{3}\right)$, and $\gamma$ is the wave frequency. We obtain:

$$
\gamma^{2}\left(\gamma^{2}-\alpha^{2}\right)-\gamma^{2} k^{2}+\alpha^{2} k^{2} \cos ^{2} \theta=0
$$

where $\theta$ is the angle between wave vector $\vec{k}$ and the $O x_{3}$ axis. Eq. (20) is the dispersion relation for plane waves in a rotating compressible fluid. We have:

$$
k=|\gamma| \sqrt{\frac{\alpha^{2}-\gamma^{2}}{\alpha^{2} \cos ^{2} \theta-\gamma^{2}}} .
$$

We can conclude that there exists a fundamental difference between the propagation of plane harmonic waves in a rotating fluid and those in a fluid at rest. From (21) with $\alpha \rightarrow 0$ we obtain $k=|\gamma|$, that is to say, the well-known relation for plane waves in a fluid at rest. We note the dependence of $k$ on the value of angle $\theta$ (the direction of the wave vector $k$ ). Two special cases are qualitatively different. In the case in which $\vec{k}$ is parallel to the rotating axis, (21) gives $k=|\gamma|$, which means that in the $O x_{3}$ direction plane waves with any frequency $\gamma$ propagate, including step-like waves $(\gamma=0)$. When we have a wave vector $\vec{k}$ with direction oblique to the rotation axis $(0<\theta<\pi / 2)$, we infer that waves propagate only for certain values of $\gamma$ : there is a forbidden zone for the frequency $\gamma$, namely $\alpha \cos \theta<\gamma<\alpha$. In the case where $\vec{k}$ is perpendicular to the rotating axis $(\theta=\pi / 2)$ we have that only waves with frequency $\gamma>\alpha$ propagate.

By differentiating the dispersion relation (20) we obtain the group velocity of the waves, thus

$$
\vec{v}_{g}=\frac{\gamma}{2 \gamma^{2}-\alpha^{2}-k^{2}}\left(k_{1}, \frac{\gamma^{2}-\alpha^{2}}{\gamma^{2}} k_{3}\right) .
$$


Meanwhile, we know that the phase velocity of the waves is

$$
\vec{c} \equiv \vec{v}_{p h}=\frac{\gamma}{k} \frac{\vec{k}}{k}=\frac{\gamma \vec{k}}{k^{2}} .
$$

From (22) we conclude the following:

(1) For the fluid at rest $(\alpha=0)$ and, hence, $k^{2}=\gamma^{2}$. We obtain

$$
\vec{v}_{\mathrm{g}}=\frac{1}{\gamma} \vec{k} \equiv \vec{v}_{\mathrm{ph}}
$$

Thus the group velocity is equal to the phase velocity; there is no dispersion, and the energy propagates in the direction $\vec{k}$.

(2) In general, for $\alpha \neq 0$, the direction of the group velocity $\vec{v}_{\mathrm{g}}$ (that is to say, the direction of propagation of the energy [12]) does not coincide with the direction of the vector $\vec{k}$, and wave dispersion takes place.

When the direction of $\vec{k}$ coincides with the rotating axis $O x_{3}$, i.e. when $\theta=0$, for the group velocity we have

$$
\vec{v}_{\mathrm{g}}=\frac{\gamma}{2 \gamma^{2}-\alpha^{2}-\gamma^{2}}\left(0, \frac{\gamma^{2}-\alpha^{2}}{\gamma^{2}} k\right)=\frac{1}{\gamma} \vec{k}=\vec{v}_{\mathrm{ph}}
$$

since $k_{1}=0, k_{3}=k$, and $k^{2}=\gamma^{2}$ in this case. Hence, the waves again propagate without dispersion.

If $\theta=\pi / 2$, and under the propagating conditions $\gamma>\alpha$, we have from (21) $k=\sqrt{\gamma^{2}-\alpha^{2}}$. Therefore, for the group velocity, we have

$$
\vec{v}_{\mathrm{g}}=\frac{\gamma}{2 \gamma^{2}-\alpha^{2}-\gamma^{2}+\alpha^{2}}(k, 0)=\frac{1}{\gamma} \vec{k} .
$$

Meanwhile from (22),

$$
\vec{v}_{\mathrm{ph}}=\frac{\gamma}{\gamma^{2}-\alpha^{2}} \vec{k}
$$

This means that $v_{g}<v_{\text {ph }}$ and, therefore, we have in this case a normal dispersion of the waves. This is in complete agreement with the fact that, for $\theta=\pi / 2$ and $k=\sqrt{\gamma^{2}-\alpha^{2}}$, the waves disperse. For $|\gamma|<\alpha$ there is a damping of waves travelling perpendicular to the $O x_{3}$ axis direction.

In the general case we can see, using (20), that the expresion $2 \gamma^{2}-\alpha^{2}-k^{2}$ in the denominator of (22) is always a positive number. From (22) it follows that 
the group-velocity vectors, corresponding to all possible plane waves starting from the same begining, are inside the limits of a characteristic cone

$$
\left|x_{3}\right|>\frac{\sqrt{\alpha^{2}-\gamma^{2}}}{\gamma}\left|x_{1}\right| .
$$

The angle between the group vector and the rotating axis $O x_{3}$ is

$$
\theta_{\mathrm{gr}}=\arctan \left[\frac{\gamma^{2}}{\gamma^{2}-\alpha^{2}} \frac{k_{1}}{k_{3}}\right]
$$

\section{Conclusion}

The general behaviour of acoustic waves in a rotating compressible fluid can be described in a simple formulation using a fourth order non-classical differential equation, which reduces to the classical one when the fluid is at rest (no rotation). The analysis of the dispersion relation exhibits the existence of a forbidden zone for the frequencies of the propagating wave. We have also seen that, in the presence of the non homogeneity of the space in which the waves propagate (owing to the rotation of the fluid), the dispersion of the waves takes place when the wave vector is not in the same direction as the rotating axis. In future work we shall study fundamental solutions of the basic equation (17) for several interesting problems involving the diffraction of waves at walls within a rotating fluid.

\section{Acknowledgments}

Partial financial support of this work under Grant Nos. GP3438 and GP249507 from the Natural Sciences and Engineering Research Council of Canada is gratefully acknowledged by two of us (respectively [RLH] and [NS]). One of us [JMA] acknowledges the hospitality of the Department of Mathematics and Statistics of Concordia University, where some of this work was carried out. 


\section{References}

[1] Yuli D. Chashechkin, International Conference on Boundary and Interior Layers (BAIL 2004), Tolouse, France, July 2004.

[2] K. Zhang, C. A. Jones. Geophysical Research Letters, 21 (1994) 1939.

[3] S. J. Friedlander. J. Geophys. J. Roy. Astro. Soc 89 (1987) 637.

[4] J. Marin-Antuna, A. Hernandez-Rodriguez, O.Sotolongo Costa. An equation for waves in a viscous rotating compressible fluid. Proc. DFD, APS, November, 1995.

[5] D. J. Acheson, Elementary Fluid Dynamics, Clarendon, Oxford (1990).

[6] K. S. Mamedov. USSR. Comput. Math. and Math. Phys, 26 (1987) 92.

[7] S. A. Gabov, K. Marin Antun'ya. USSR. Comput. Math. and Math. Phys, 25 (1986) 873.

[8] S.A. Gabov. Diffraction of internal waves that are described by the Klein-Gordon equation, on the half plane. Dokl. Akad. Nauk SSSR, 264 (1982) 73.

[9] S.A. Gabov, A.G. Sveshnikov, and A.K. Shatov. Asymptotic behavior of the solution of the problem of waves on the surface of a thin spherical layer of stratified liquid in the presence of obstacles, Dokl. Akad. Nauk SSSR, 268 (1983) 1095.

[10] S. A. Gabov and J. Marín-Antuña. Vestnik Mosk. Univ.-3, 26 (1985) 16.

[11] L.M. Brekhovskikh and V. Goncharov. Mechanics of Continua and Wave Dynamics (Springer Series on Wave Phenomena, No 1) Springer-Verlag, New York, 2nd edition (1994).

[12] G. B. Whitham. Linear and Nonlinear Waves, Wiley-Interscience, New York (1999).

[13] L.D. Landau and E.M. Lifshitz. Fluid Mechanics. Butterworth-Heinemann, 2nd edition (1987).

[14] V. N. Maslennikova. Private Communication (Summary of doctoral dissertation, Novosibirsk, 1971).

[15] S. L. Sobolev. On a new problem of Mathematica Physics. An. Rom. - Sov. Sr. Mat. Fiz. 9 (1955) 5. 